\title{
Glucose metabolism regulation in morbidly obese patients and in patients after biliopancreatic diversion
}

\section{Troshina E.A., Mazurina N.V., Ogneva N.A., Yashkov Y.I.*}

\author{
Endocrinology Research Center, Moscow, Russia
}

*Endosurgery and Lithotripsy Center, Moscow, Russia.

Introduction: Morbid obesity (MO) is associated with high frequency of type 2 diabetes mellitus (T2DM). Biliopancreatic diversion (BPD) is bariatric operation that results in rapid T2DM remission and increased GLP-1 levels. The aim of the study was to compare nondiabetic MO patients with normal weight controls and with patients who underwent BPD more than 2 years ago.

Methods: Blood glucose levels, IRI, GLP-1, GIP and glucagon were measured during the oral glucose tolerance test (OGTT) in three groups of patients. Patients of the 1st group (MO) had BMI> $40(\mathrm{n}=$ 22) and no history of diabetes mellitus. Patients after BPD were included in the 2 nd group ( $n=23$ ), post operative period median was 4.7 years (2.3-7.2). The 3rd group were normal weight controls $(n=$ 22).

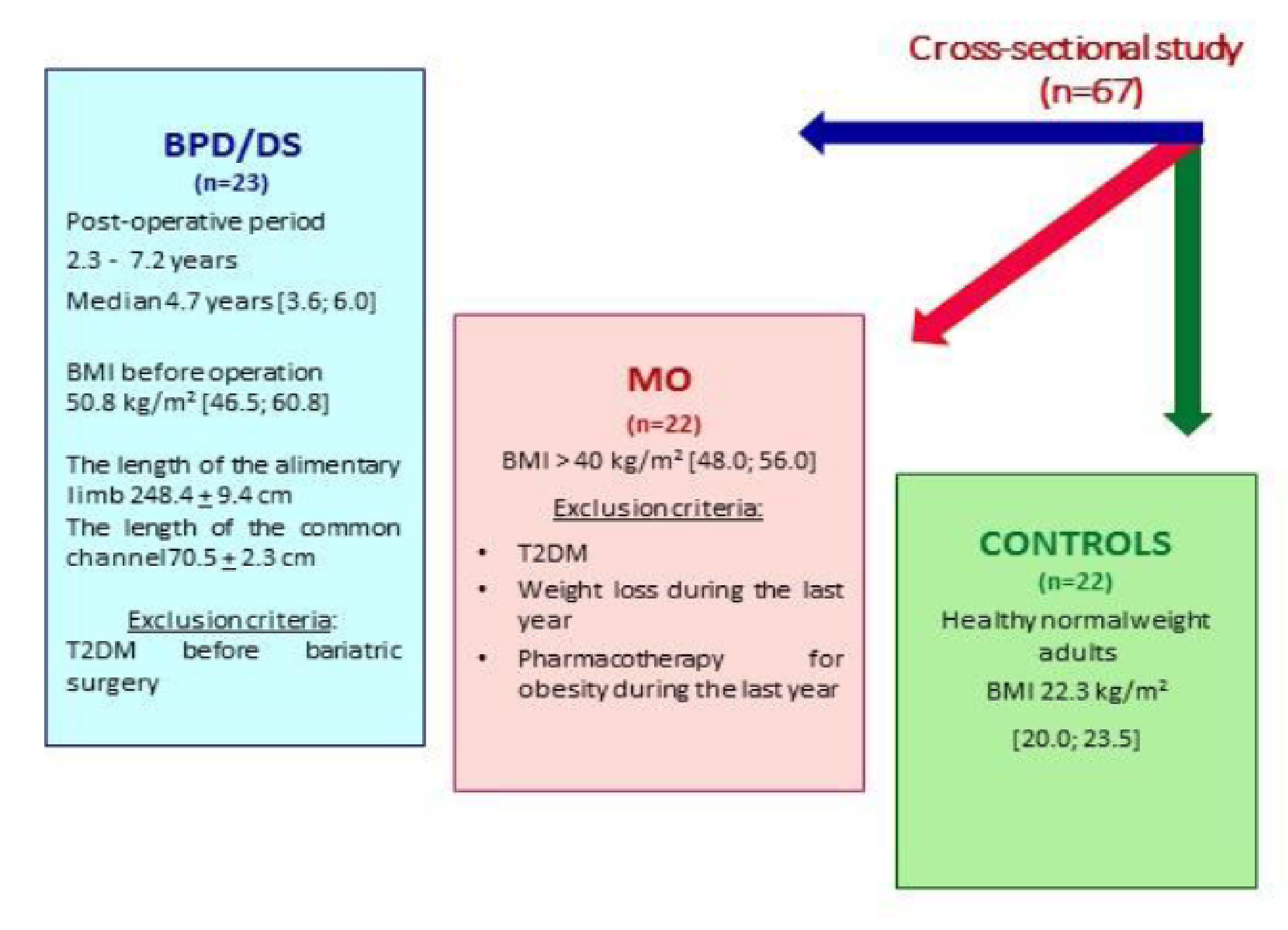

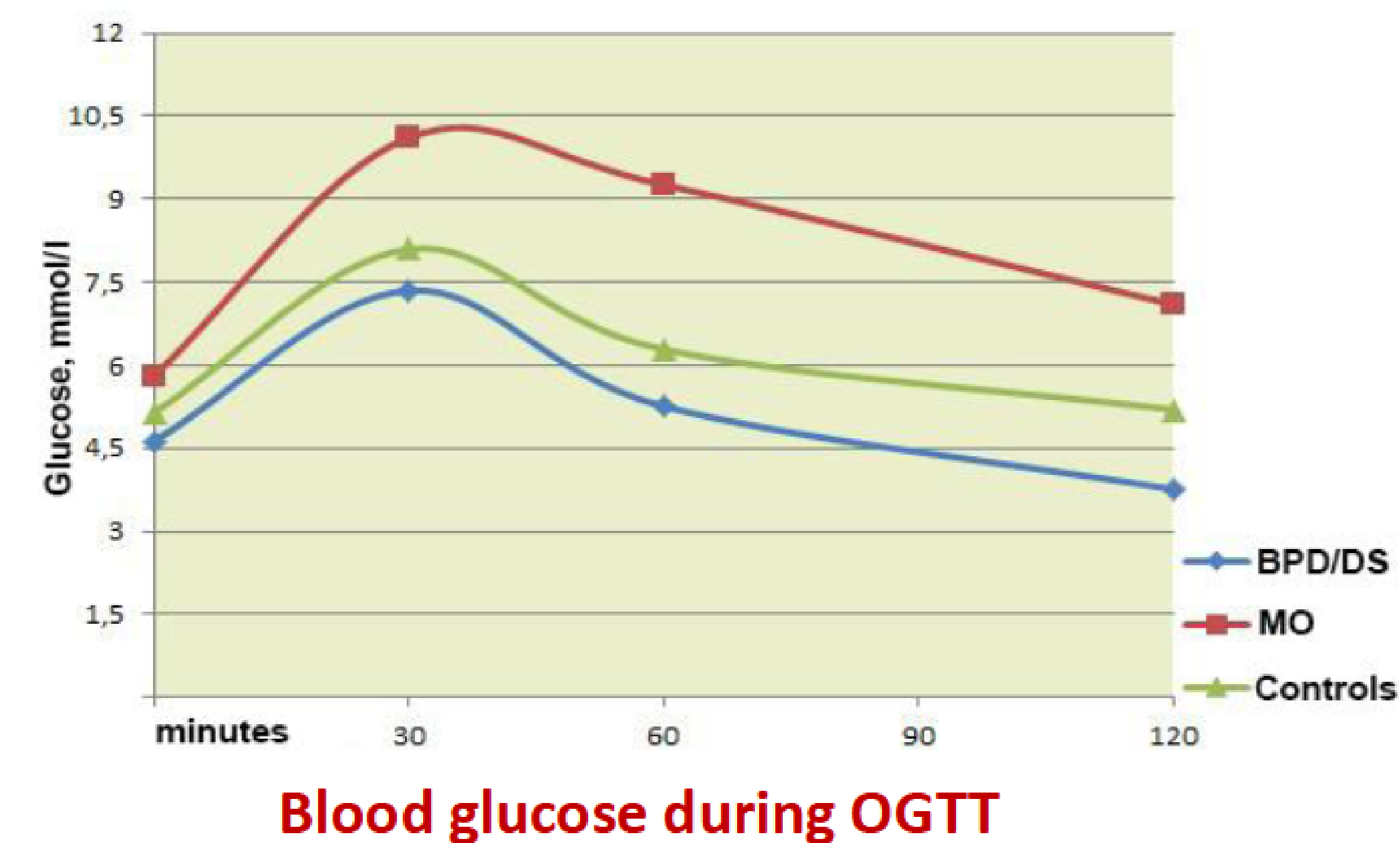

Blood glucose during OGTT

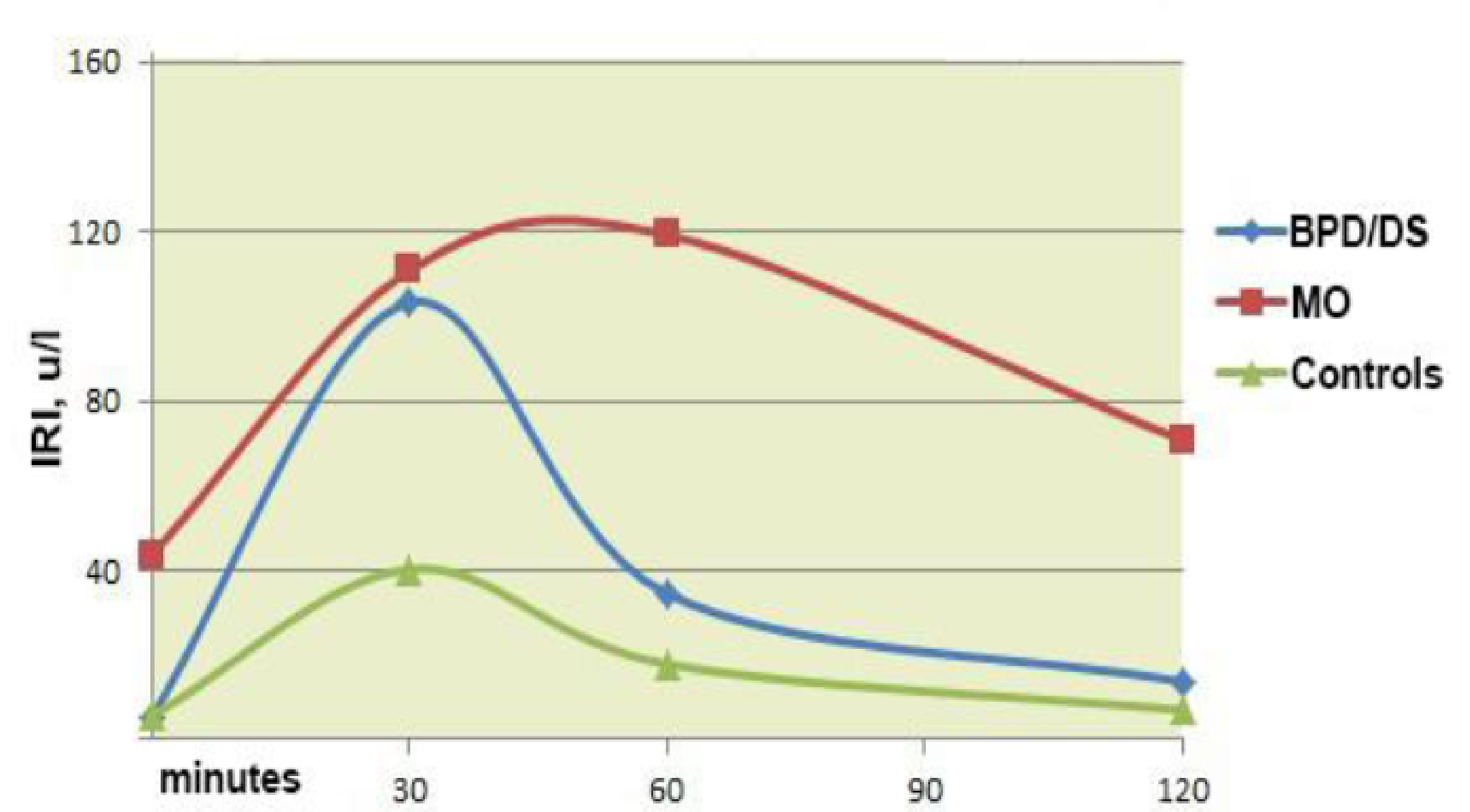

Insulin levels during OGTT

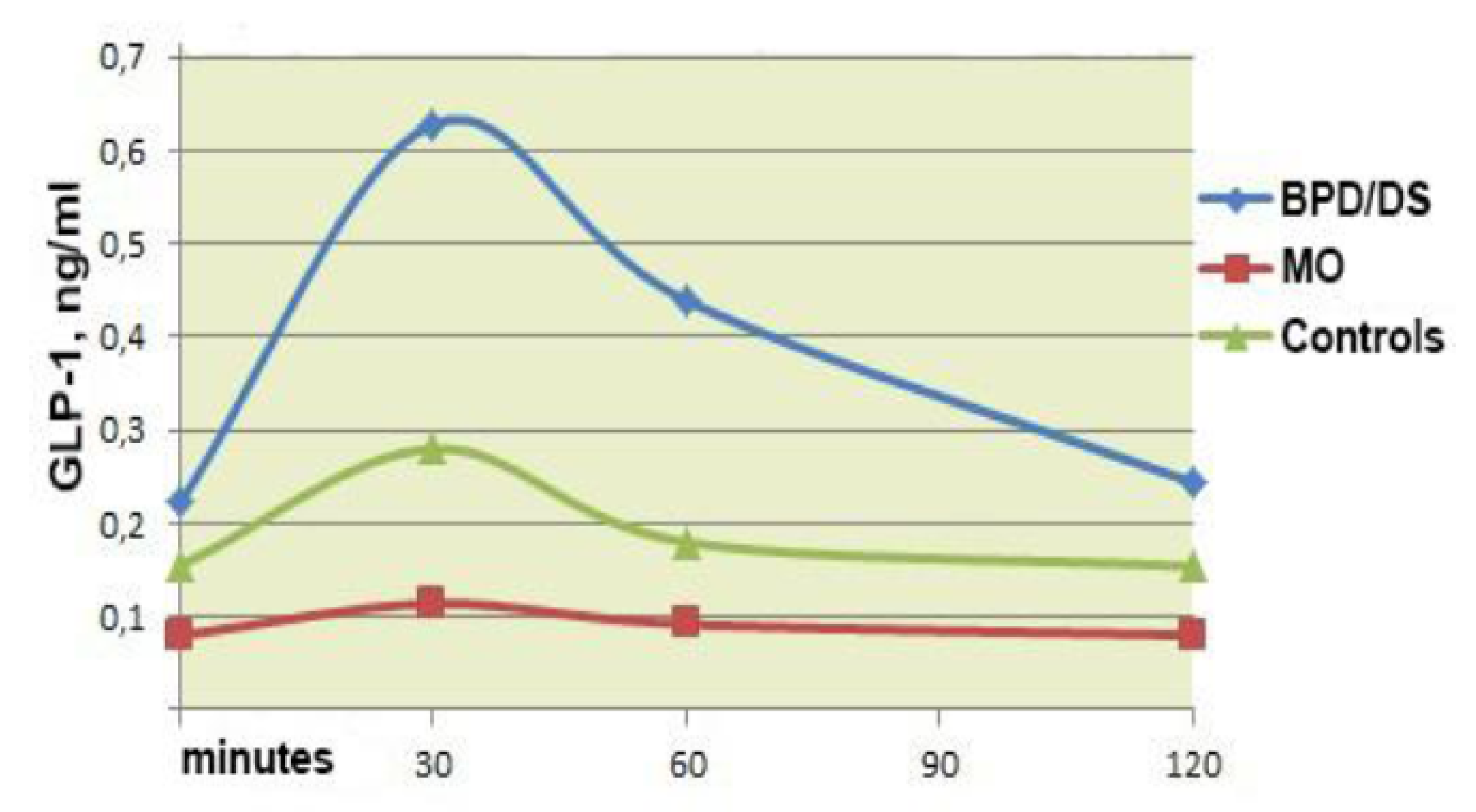

GLP-1 levels during OGTT

Results: Impaired glucose metabolism was revealed in $68.2 \%$ of $\mathrm{MO}$ patients $(n=10)$.In MO group fasting glucose, IRI and HOMA-IR were maximal $(p<0.001)$. MO patients had higher fasting and stimulated GIP and glucagon levels. In the BPD patients postprandial glucose (120 min) was lower, in $17.4 \%$ we found postprandial hypoglycemia ( $<2.8$ mmoIVI). Stimulated IRI concentration was significantly higher in the BPD group ( $p=0.026)$. Fasting and stimulated GLP-1 were

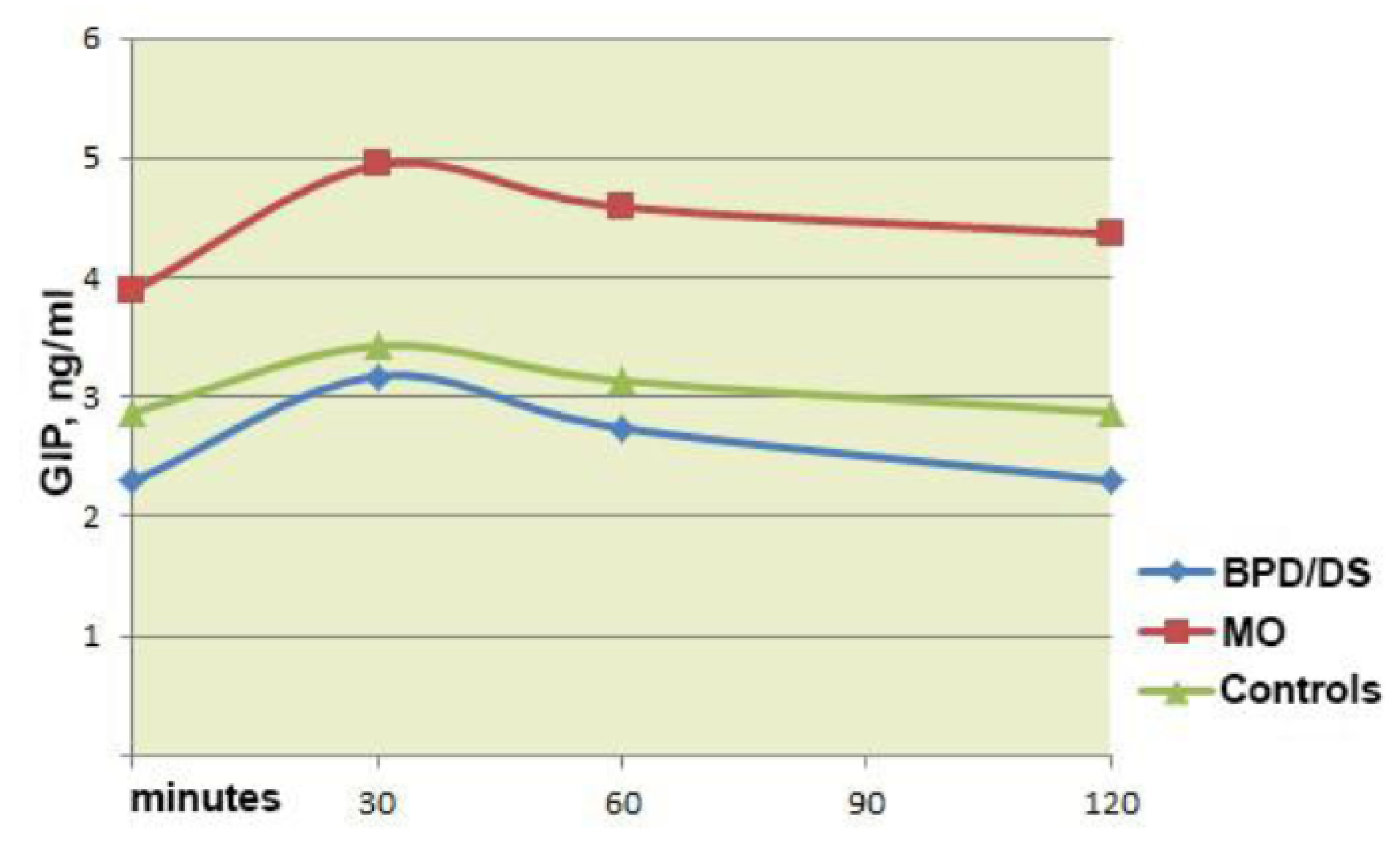

GIP levels during OGTT

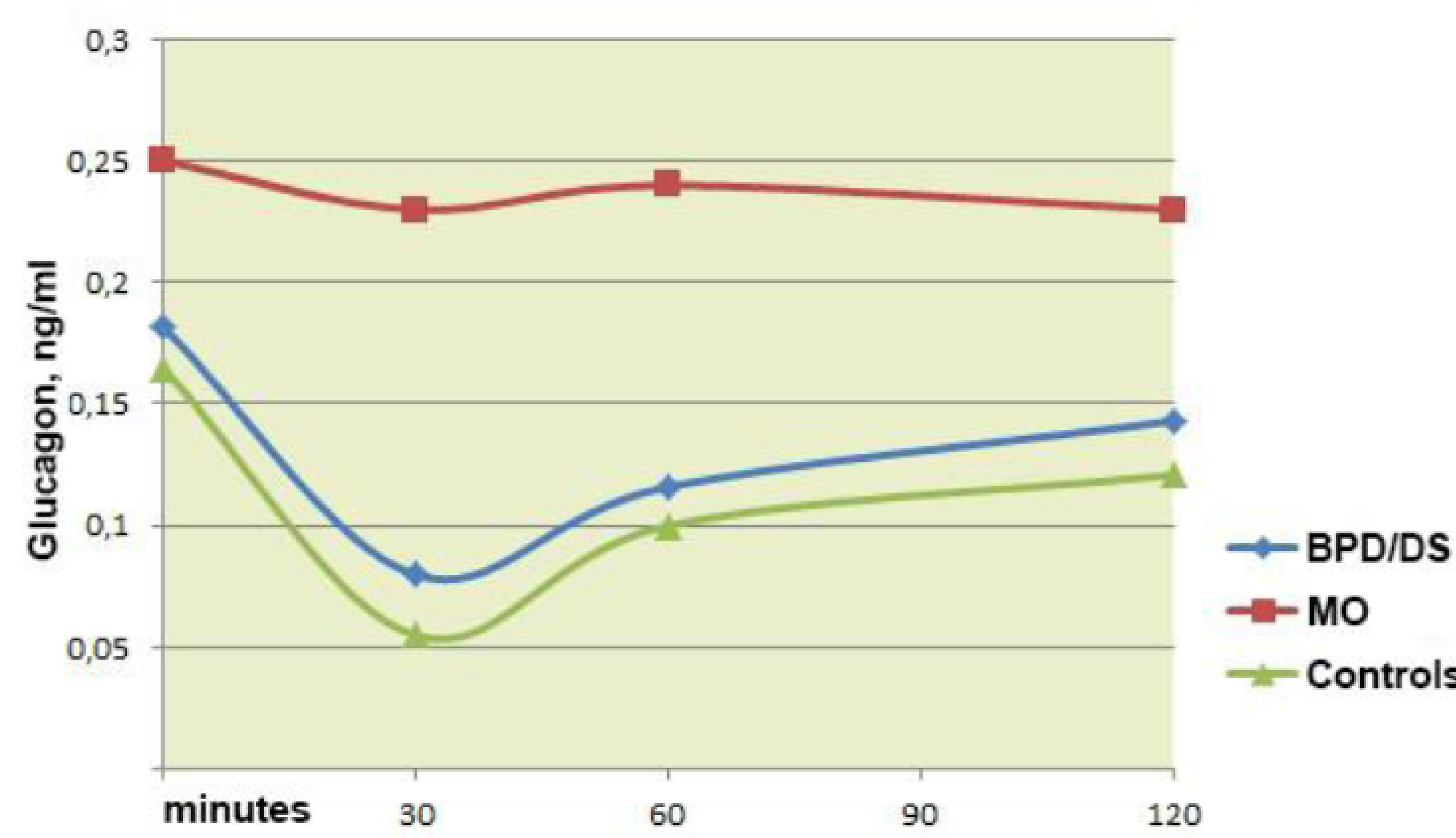

Glucagon levels during OGTT

Conclusion: Hyperglucagonemia, increased GIP levels and decreased GLP-1 levels are observed in MO. Glucose intolerance and insulin resistance incidence is higher in MO patients. Stimulated plasma IRI and GLP concentrations are significantly increased in BPD patients. 